University of South Carolina

Scholar Commons

$11-1-2005$

\title{
Effects of EBG Reflection Phase Profiles on the Input Impedance and Bandwidth of Ultrathin Directional Dipoles
}

\author{
M. Faisal Abedin \\ University of South Carolina - Columbia \\ Mohammod Ali \\ University of South Carolina - Columbia, alimo@engr.sc.edu
}

Follow this and additional works at: https://scholarcommons.sc.edu/elct_facpub

Part of the Electrical and Computer Engineering Commons

\footnotetext{
Publication Info

Published in IEEE Transactions on Antennas and Propagation, Volume 53, 2005, pages 3664-3672. http://ieeexplore.ieee.org/xpl/Recentlssue.jsp?punumber=8 (c) 2005 by IEEE
}

This Article is brought to you by the Electrical Engineering, Department of at Scholar Commons. It has been accepted for inclusion in Faculty Publications by an authorized administrator of Scholar Commons. For more information, please contact digres@mailbox.sc.edu. 


\title{
Effects of EBG Reflection Phase Profiles on the Input Impedance and Bandwidth of Ultrathin Directional Dipoles
}

\author{
M. Faisal Abedin, Student Member, IEEE, and Mohammod Ali, Senior Member, IEEE
}

\begin{abstract}
A comprehensive study of the effects of the reflection phase profiles on dipole antennas is presented with the ultimate objective of designing ultrathin printed dipoles (as thin as one hundredth of the wavelength). Dipole driving-point impedance and bandwidth are studied as function of various electromagnetic bandgap (EBG) reflection phase profiles. It is demonstrated that although many different reflection phase profiles can be generated for a certain antenna height, it is the profile that satisfies a specific range of reflection phase angles that is required to achieve good antenna performance. Such optimum EBG phase profiles are generated for a number of antenna heights and their influence on a dipole antenna impedance and bandwidth are also studied. Finally, an actual EBG structure and a printed dipole antenna are designed, fabricated and tested. The overall antenna height for this case was $0.03 \lambda$. The computed and measured results show that efficient printed dipoles on ultrathin grounded dielectric substrates can be developed that will substantially reduce the sizes and weight of large arrays.
\end{abstract}

Index Terms-electromagnetic bandgap (EBG), input impedance, low-profile, reflection phase.

\section{INTRODUCTION}

$\mathbf{L}$ OW-PROFILE printed dipole and slot antenna elements are widely used in large arrays on ground-based, vehicular, air-borne, and ship-board applications. To achieve a unidirectional beam such elements are operated against a metallic ground plane. An arrangement is shown in Fig. 1(a). The radiation beam is restricted to the upper hemisphere and can be scanned by employing the appropriate phase shift in each individual radiating element.

However, since linear elements, such as dipoles suffer from serious performance degradation when operated very close to a ground plane an arrangement shown in Fig. 1(b) is usually used [1]. This ensures enough separation of such elements from the ground plane. Needless to say, this arrangement is obtrusive, three dimensional, and expensive due to material cost and fabrication difficulty. The performance degradation for the arrangement shown in Fig. 1(a) results due to two reasons: (a) given the radiating elements close proximity to the metallic ground plane, the reflected waves add destructively with the incident radiation and (b) surface wave mode of radiation results in antenna efficiency degradation. The latter one aggravates with increasing substrate thickness, dielectric constant, and frequency. Clearly

Manuscript received May 25, 2004; revised June 28, 2005.

The authors are with the Department of Electrical Engineering, University of South Carolina, Columbia, SC 29208 USA (e-mail: alimo@engr.sc.edu).

Digital Object Identifier 10.1109/TAP.2005.858584

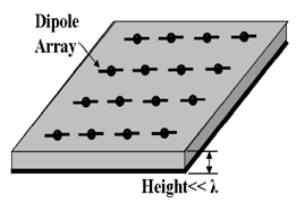

(a)

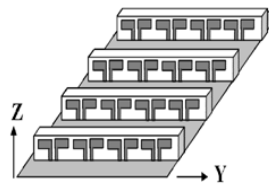

(b)

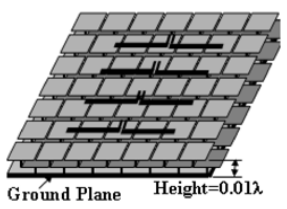

(c)
Fig. 1. Array of printed dipoles on (a) thin grounded dielectric substrate, (b) dielectric-sheets normal to the ground plane, and (c) proposed EBG structure backed by ground plane.

an extremely thin low-profile printed dipole array will be greatly desirable both from performance and cost requirements.

Such a problem with printed dipole arrays on top of thin grounded dielectric substrates [see Fig. 1(a)] may be solved by employing engineered materials [2]. For instance, an engineered electromagnetic bandgap (EBG) structure can be placed in between the antenna and the ground plane layer. Since an EBG structure does not reflect back the incident wave with $180^{\circ}$ phase reversal a linear antenna element, such as a dipole can be placed very close to it.

To date researchers have primarily focused on EBG development and their integration with antennas for surface wave suppression [3]-[15]. The topic of low-profile linear antenna development has drawn some interest recently [16]-[21]. In [16] a comparative overview of PBG, PMC, and perfect electric conducting (PEC) structures was presented. A low-profile cavity backed slot antenna on a uniplanar UC-PBG structure was proposed in [17]. Similarly, a low-profile circularly polarized patch antenna was proposed in [18]. Hansen [19] first introduced an analytical representation of the effects of a high impedance screen on a half-wavelength dipole antenna. He identified $\pm 45^{\circ}$ as the useful range of reflection phase angles for efficient dipole operation. In [20] effects of a PBG structure on the performance of a planar inverted-F antenna have been studied. Recently further studies of the reflection phase characteristics of a mushroom type EBG structure have revealed that the $\mathrm{EBG}$ ground plane requires a reflection phase in the range of $90^{\circ} \pm 45^{\circ}$ for a low profile wire antenna to obtain a good return loss [21]. The overall height of the EBG structure in conjunction with the dipole antenna proposed was $0.06 \lambda$. The study presented in [21] considered determining the suitable reflection phase angles by varying the length of the dipole antenna and observing the antenna return loss characteristics. In contrast, the objective of this paper is to investigate the effect of the EBG reflection phase characteristics on the driving-point impedance of a dipole antenna. To this end, the well-established 
concepts of driven and image dipole and self and mutual impedances are re-introduced and used in the context of an EBG structure, suitable positive and negative reflection phase angles are identified for dipole antennas at different heights, a practical EBG structure design guideline is provided, an EBG structure along with a printed thin dipole antenna is designed and tested to validate performance. Interestingly it is also observed that as the reflection phase of an EBG structure changes with frequency from positive to negative values the dipole antenna on top of it exhibits a distinct dual-band characteristic.

The paper is organized as follows. First, hypothetical EBG structures with various constant reflection phase profiles with frequency are considered. The effects of such reflection phase profiles on the driving-point impedance and VSWR bandwidth of a dipole antenna are investigated. From this study, ranges of reflection phase angles are identified for various antenna heights. Second, since reflection phase profiles of real EBG structures vary from $180^{\circ}$ to $-180^{\circ}$ with frequency the effect of various sample phase profiles on a dipole are investigated. It is demonstrated that for a specific antenna height the reflection phase profile that contains the useful phase angles identified in step 1 results in the best bandwidth performance. Third, based on the existing EBG design guidelines a three-layer optimum EBG structure is designed that reflects our findings in step 2. Such a structure is analyzed along with a printed dipole above it using IE3D. Finally, the proposed EBG structure and the dipole antenna are fabricated and tested to demonstrate performance. In this paper the term "stopband frequency" is defined as the frequency corresponding to the $0^{\circ}$ reflection phase angle.

\section{INPUT IMPEDANCE OF A THIN WIRE DiPOLE ANTENNA}

\section{A. Dipole Impedance in Free Space and Against a Ground Plane (PEC)}

For a dipole antenna operating against a ground plane at a height, $h$ the driving-point impedance consists of the dipole's self and mutual impedances, where the latter is computed by removing the ground plane and constructing an image dipole on the opposite side of the ground plane at a distance, $2 h$ from the primary dipole.

The self-impedance of a thin-wire dipole (derived using the induced EMF method) can be expressed as [22]

$$
\begin{aligned}
Z_{11}= & -\frac{1}{\sin ^{2}\left(\frac{k l}{2}\right)} \\
& \times \int_{-l / 2}^{l / 2} \sin \left[k\left(\frac{l}{2}-\left|z^{\prime}\right|\right)\right] E_{z}\left(\rho=a, z=z^{\prime}\right) d z^{\prime}
\end{aligned}
$$

where $E_{z}$ is the electric field radiated by the dipole (length $l$ ) and is given by

$$
\begin{aligned}
E_{z} & =-j \frac{\eta I_{0}}{4 \pi}\left[\frac{e^{-j k R_{1}}}{R_{1}}+\frac{e^{-j k R_{2}}}{R_{2}}-2 \cos \left(\frac{k l}{2}\right) \frac{e^{-j k r}}{r}\right] \\
r & =\sqrt{\rho^{2}+z^{2}}, \quad R_{1}=\sqrt{\rho^{2}+\left(z-\frac{l}{2}\right)^{2}} \\
R_{2} & =\sqrt{\rho^{2}+\left(z+\frac{l}{2}\right)^{2}}
\end{aligned}
$$

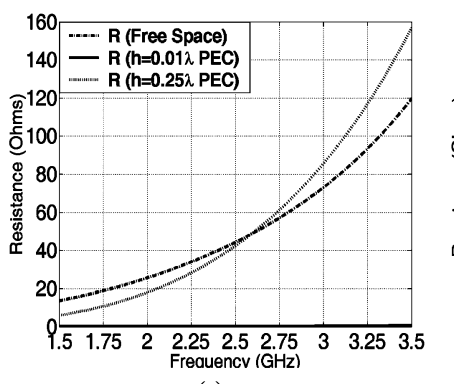

(c)

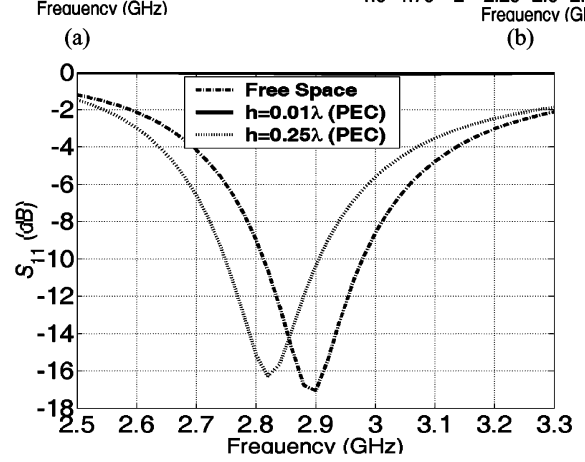

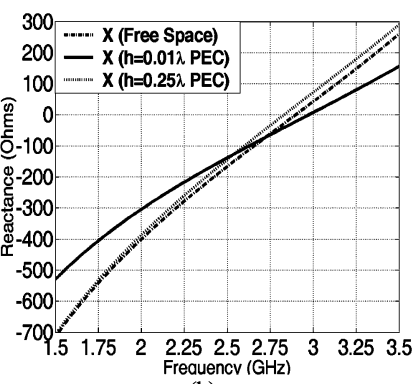

(b)
Fig. 2. Computed input (a) resistance, (b) reactance, and (c) corresponding S11 (dB) data of a thin wire dipole.

where the symbols have their usual meanings. Since the integral in (1) does not have a closed-form solution a numerical MATLAB integration routine was used. The mutual impedance is given by [23]

$$
\begin{aligned}
Z_{12}= & \frac{j 30}{\sin k l_{1} \sin k l_{2}} \\
& \times \int_{-l_{2}}^{l_{2}}\left(\frac{e^{-j k r_{1}}}{r_{1}}+\frac{e^{-j k r_{2}}}{r_{2}}-2 \cos k l_{1} \frac{e^{-j k r}}{r}\right) \\
& \times \sin k\left(l_{2}-\left|\zeta_{2}\right|\right) d \zeta_{2} \\
r= & {\left[y^{2}+\left(z+\zeta_{2}\right)^{2}\right]^{1 / 2} } \\
r_{1}= & {\left[y^{2}+\left(z+\zeta_{2}-l_{1}\right)^{2}\right]^{1 / 2} } \\
r_{2}= & {\left[y^{2}+\left(z+\zeta_{2}+l_{1}\right)^{2}\right]^{1 / 2} }
\end{aligned}
$$

where the dipole length is $2 l$ and $l_{1} l_{2}=l$.

The driving-point impedance is given by

$$
Z_{d}=Z_{11}+Z_{12} e^{j \theta}
$$

where $\theta$ is the phase angle of the reflected wave, which is $180^{\circ}$ for a PEC. Fig. 2 shows the computed driving point impedance of a thin wire dipole of length $50 \mathrm{~mm}$ and radius $0.01 \mu \mathrm{m}$. For comparison, the impedance at antenna heights of $0.01 \lambda$ and $0.25 \lambda$ ( $\lambda$ corresponds to the dipole resonant frequency in free space) from a PEC are also shown. The impedance data for $h=0.25 \lambda$ and that in free space are similar because the mutual coupling between the driven and image dipoles is negligible due to the two times quarter wavelength distance. The return loss data shown in Fig. 2(c) (referred to a $50 \Omega$ feed) further corroborates this. For $h=0.01 \lambda$ the driving-point resistance is very small due to the strong mutual coupling between the dipole and its image. This can be understood from the mutual impedance data of the dipole for $h=0.01 \lambda$ plotted in Fig. 3. For $h=0.01 \lambda$ the mutual resistance is close to the self resistance [shown in 


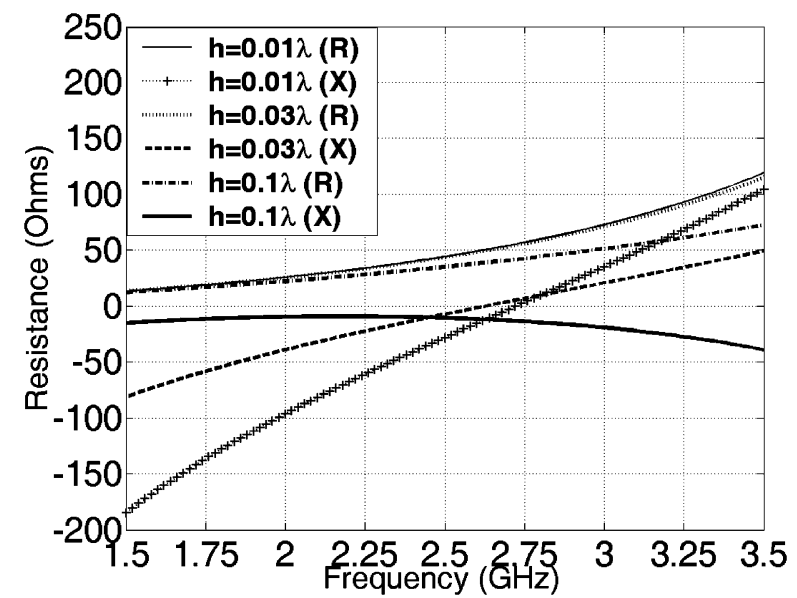

Fig. 3. Computed mutual impedance of a thin wire dipole placed at different heights from the ground plane.

Fig. 2(a)], which results in negligible driving-point resistance $\left(R_{d}\right)$ and poor return loss as shown in Fig. 2(c).

\section{B. Dipole Impedance Against a Constant Reflection Phase EBG Surface}

1) Antenna Height $=0.01 \lambda$ : For an EBG surface with various reflection phase characteristics the driving-point impedance of a dipole antenna can similarly be calculated using (4). We assume that an EBG structure has been inserted in between the ground plane and the printed dipole that provides the appropriate reflection phase needed for proper operation. For simplicity of analysis we consider a hypothetical EBG surface that can generate a constant reflection phase, $\theta$ with frequency. In contrast, $Z_{11}$ and $Z_{12}$ in (4) are functions of frequency. For a thin wire dipole, placed at different heights from a ground plane $Z_{d}$ was computed as a function of frequency with $\theta$ as the parameter. Note that $Z_{11}$ is the self-impedance of the dipole indicated as "free-space" in Fig. 2(a) and (b). The mutual impedance for the dipole at different heights as computed using (3) are shown in Fig. 3. It is understandable that for a real EBG surface it may be difficult to keep $\theta$ constant with frequency. However, an analytical study with a constant $\theta$ can clearly demonstrate what specific phase angles have stronger influence on $Z_{d}$.

Computed $Z_{d}$ data for a dipole placed at a height of $0.01 \lambda$ from the ground plane for different reflection phase angles are shown in Fig. 4. When $\theta$ is negative the driving point resistance [Fig. 4(a)] is negative at low frequencies because the large mutual resistance resulting from strong mutual coupling adds destructively with the small self-resistance. In contrast, when $\theta$ is positive the larger mutual resistance adds constructively with the self-resistance. Observing the reactance characteristics in Fig. 4(b) it is clear that the antenna is resonant at two different frequencies for positive and negative $\theta$, respectively. The resonant frequency is around $2.75 \mathrm{GHz}$ for positive $\theta$ and $3.1 \mathrm{GHz}$ for negative $\theta$. Thus, for a real EBG surface where $\theta$ varies from positive to negative distinct dual-band performance can be obtained. This is evident from the VSWR characteristics shown in Fig. 4(c). These curves were generated by computing the reflection coefficient between the dipole driving-point impedance and a $50 \Omega$ feed transmission line.

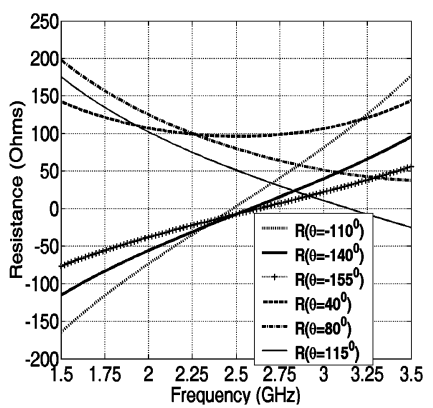

(a)

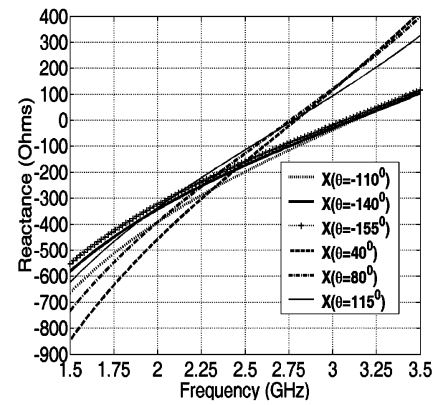

(b)

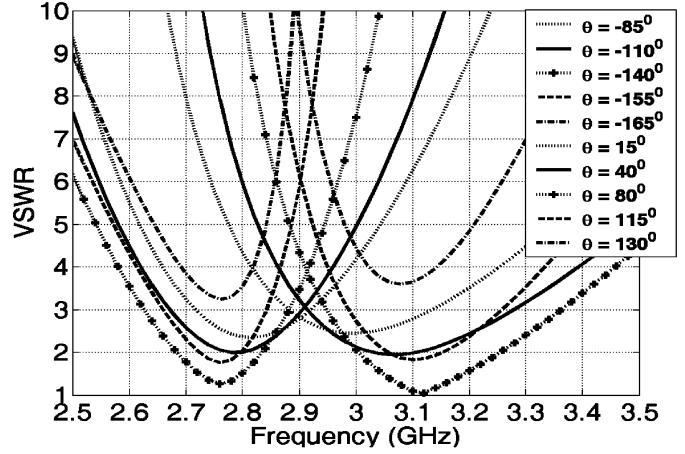

(c)

Fig. 4. Computed driving point (a) resistance, (b) reactance and (c) VSWR data of a thin wire dipole for various hypothetical EBG reflection phase angles. Antenna height $=0.01 \lambda$ from the ground plane.

It is clear that for positive reflection phase angles the antenna has a VSWR minimum at around $2.75 \mathrm{GHz}$ while that for negative reflection phase angles it is at around 3.1 GHz. We also inspect the reflection phase angles for which the VSWR data around these two frequency bands are within 2:1. It is apparent that for $\theta$ varying between $40^{\circ}$ to $115^{\circ}$ (from 2.68 to 2.84 $\mathrm{GHz}$ ) and from $-110^{\circ}$ to $-155^{\circ}$ (from 3 to $3.25 \mathrm{GHz}$ ) result in VSWR values less than or equal to 2 . We define these phase angles as useful phase angles since they provide good VSWR performance for the dipole antenna. Interestingly the ordinarily narrowband (6\%) thin wire dipole demonstrates strong potential for dual band operation with considerable bandwidth $5.8 \%$ and 8.0\% within 2:1 VSWR) for each band, when an EBG surface is placed in between the ground plane and the dipole.

2) Antenna Height $=0.03 \lambda$ : Computed data for this case are shown in Fig. 5. Comparing Figs. 4(a) and 5(a), it is clear that for negative phase angles the driving point resistance for the latter is mostly positive. This is because the smaller mutual resistance is dominated by the self-resistance due to increased antenna height. As before the reactance data [Fig. 5(b)] shows the sign of dual resonance. Comparing Figs. 4(b) and 5(b) it is seen that the two resonant frequencies $(2.76$ and $3.1 \mathrm{GHz})$ for the latter have moved somewhat closer to each other. From Fig. 5(c), the useful ranges of phase angles are $40^{\circ}$ to $115^{\circ}$ (from 2.68 to $2.84 \mathrm{GHz}$ ) and $-95^{\circ}$ to $-145^{\circ}$ (from 2.97 to $3.2 \mathrm{GHz}$ ). Note that the ranges of useful phase angles for a dipole height of $0.01 \lambda$ are $40^{\circ}$ to $115^{\circ}$ and $-110^{\circ}$ to $-155^{\circ}$. Thus, a small increase in the dipole height did not change the range of positive phase angles while the range of negative phase angles is widened by $5^{\circ}$.

3) Antenna Height $=0.1 \lambda$ : To further investigate the relationship between the EBG reflection phase characteristics and 


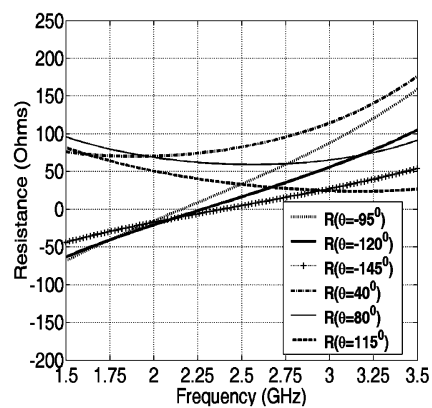

(a)

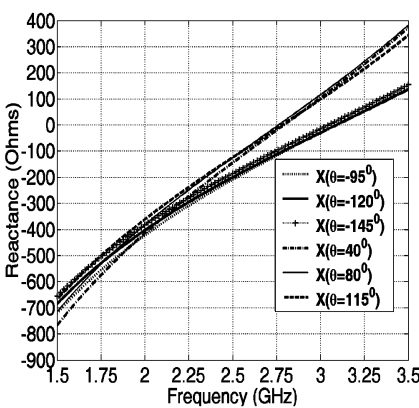

(b)

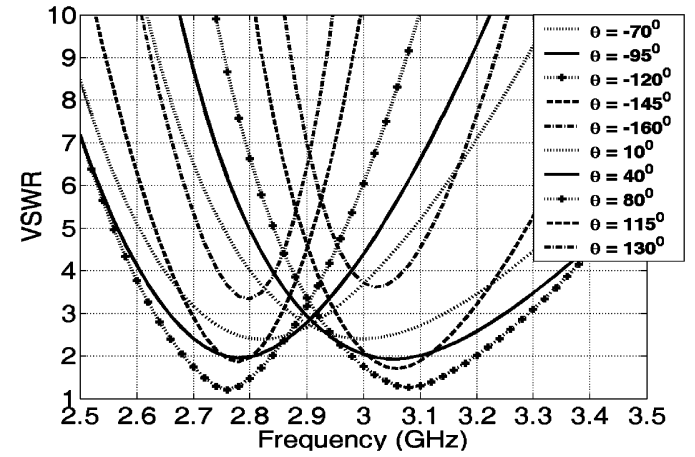

(c)

Fig. 5. Computed driving point (a) resistance, (b) reactance and (c) VSWR data of a thin wire dipole for various hypothetical EBG reflection phase angles. Antenna height $=0.03 \lambda$ from the ground plane.

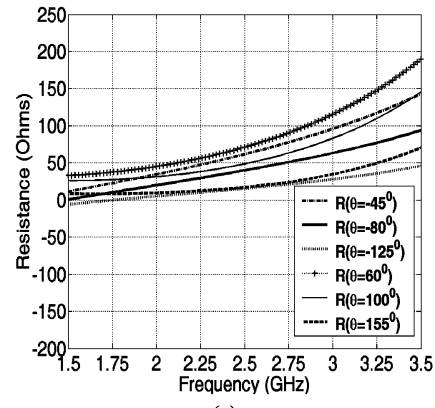

(a)

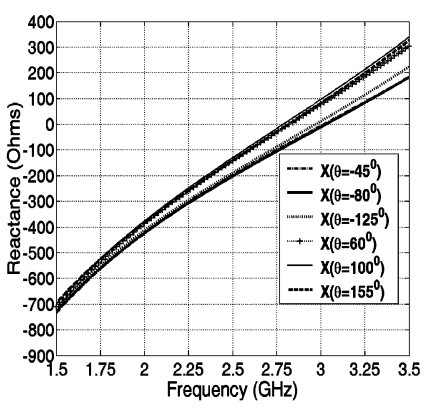

(b)

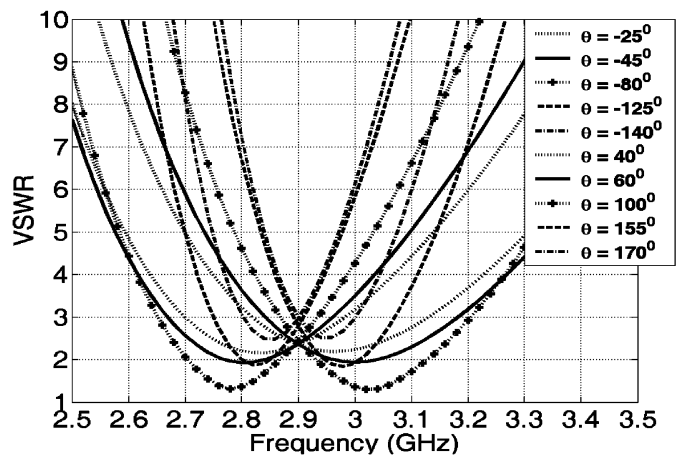

(c)

Fig. 6. Computed driving point (a) resistance, (b) reactance and (c) VSWR data of a thin wire dipole for various hypothetical EBG reflection phase angles. Antenna height $=0.1 \lambda$ from the ground plane.

the performance of a dipole we consider an antenna height of $0.1 \lambda$ [see Fig. 6]. Within the frequency range of interest the resistance for all values of $\theta$ is positive as because $Z_{12}$ is small and hence dominated by $Z_{11}$. Observing the reactance and VSWR plots shown in Fig. 6(b) and 6(c) it is clear that the two reso-

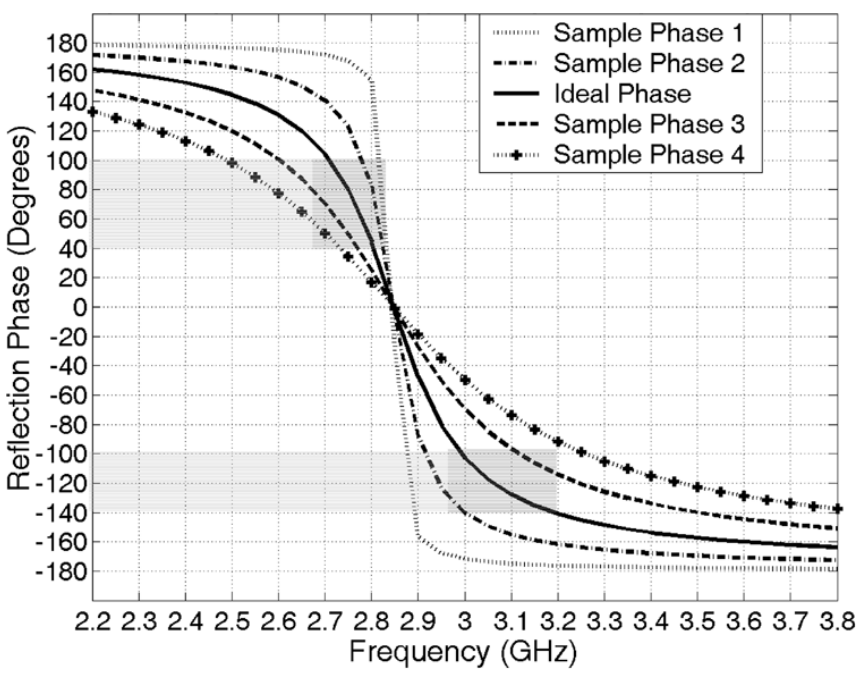

Fig. 7. Identifying the optimum reflection phase profile of an EBG structure for a thin-wire dipole antenna placed at a height of $0.03 \lambda$ from the ground plane.

nant frequencies (2.77 and 3.0 GHz) have moved even closer to each other. The useful ranges of phase angles are $60^{\circ}$ to $155^{\circ}$ (from 2.7 to $2.87 \mathrm{GHz}$ ) and $-45^{\circ}$ to $-125^{\circ}$ (from 2.93 to 3.13 $\mathrm{GHz}$ ). Further investigation showed that for antenna height of $0.2 \lambda$ the two resonant frequencies merged with each other and the dual-band characteristics disappeared.

\section{Dipole Impedance Against a Real EBG Surface}

The reflection phase, $\theta$ of an EBG structure varies continuously from $180^{\circ}$ to $-180^{\circ}$ in the vicinity of its stopband and is given by [2]

$$
\theta(f)=\operatorname{Im}\left[\ln \left(\frac{a f^{2}+j b f-c}{-a f^{2}+j b f+c}\right)\right]
$$

where $a, b$, and $c$ are the EBG design parameters with $a=$ $4 \pi^{2} \eta L C, b=2 \pi L, c=\eta$. Here $L$ and $C$ represent typical sheet inductance and capacitance of the EBG structure and $\eta$ stands for the intrinsic impedance of free-space. The profile of the reflection phase versus frequency curve can be changed by varying the design parameters $a$ and $b$ ( $c$ is constant). Since $a$ and $b$ are directly related to the actual inductance, $L$ and capacitance, $C$ provided by an EBG structure such a structure can be designed using the formulas available in [2]. This is discussed in more details in the latter part of this section.

Fig. 7 shows the reflection phase versus frequency curves for five different EBG structures. These curves were generated by varying parameter $b$, while keeping $a$ constant as indicated in Table I. The inductance and capacitance values corresponding to the parameters $a$ and $b$ depend on the actual EBG geometry and material characteristics. From Fig. 7 it is clear that all the EBG structures have the same stopband frequency defined as the frequency corresponding to the $0^{\circ}$ phase angle. As will be illustrated in Sections III and IV the variation of the reflection phase profile with frequency affects the antenna impedance, bandwidth and gain. Therefore, just by making the reflection phase stopband frequency of the EBG structure coincide with the antenna resonant frequency is not enough to insure efficient operation. The reflection phase curve indicated "ideal phase" represents the useful phase angles required for efficient dual-band 
TABLE I

Values of EbG Design Parameters, Inductances and Capacitances of Different Reflection Phase Curves Based on a Dipole OPERATING AT $0.03 \lambda$ HeIGHT From THE GROUND

\begin{tabular}{|c|c|c|c|c|c|}
\hline $\begin{array}{c}\text { Reflection } \\
\text { Phase Types }\end{array}$ & $\begin{array}{c}\text { Design } \\
\text { parameter } \\
\boldsymbol{a \times 1 0 ^ { - 1 7 }}\end{array}$ & $\begin{array}{c}\text { Design } \\
\text { parameter } \\
\boldsymbol{b \times 1 0 ^ { - 8 }}\end{array}$ & $\begin{array}{c}\text { Inductance } \\
\boldsymbol{L}(\mathbf{n H})\end{array}$ & $\begin{array}{c}\text { Capacitance } \\
\boldsymbol{C} \\
(\mathbf{p F}-\mathbf{s q u a r e})\end{array}$ & $\begin{array}{c}\text { Stop-band } \\
\text { Frequency } \\
\boldsymbol{f}_{\boldsymbol{c}}(\mathbf{G H z})\end{array}$ \\
\hline Sample Phase1 & 4.65 & 0.1 & 0.16 & 19.6 & 2.85 \\
\hline Sample Phase2 & 4.65 & 0.5 & 0.8 & 3.93 & 2.85 \\
\hline Ideal Phase & 4.65 & 1.1 & 1.75 & 1.78 & 2.85 \\
\hline Sample Phase3 & 4.65 & 2.0 & 3.18 & 0.98 & 2.85 \\
\hline Sample Phase4 & 4.65 & 3.0 & 4.77 & 0.65 & 2.85 \\
\hline
\end{tabular}

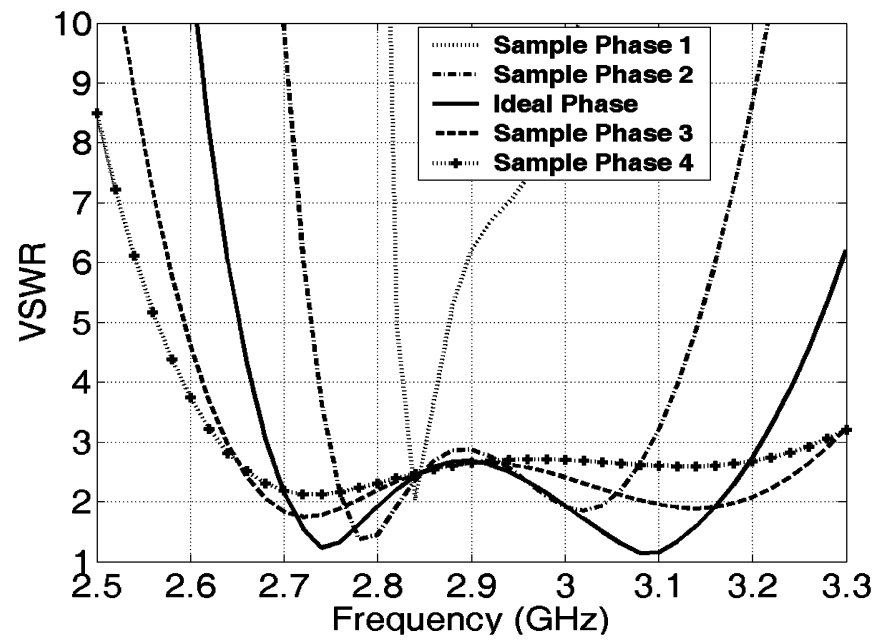

Fig. 8. Corresponding VSWR data of the thin-wire dipole.

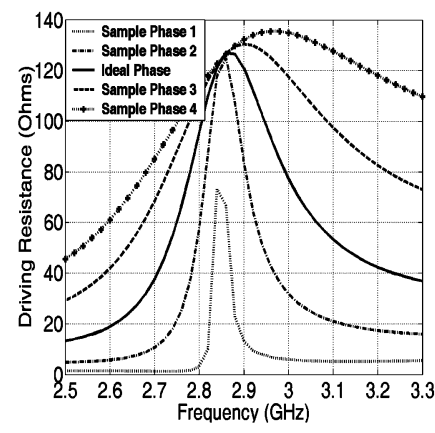

(a)

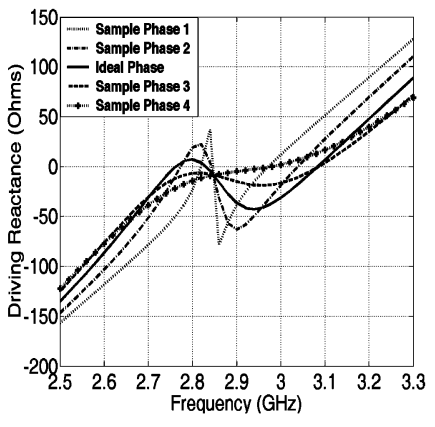

(b)
Fig. 9. Computed driving-point (a) resistance and (b) reactance of a thin-wire dipole placed at a height of $0.03 \lambda$ from EBG surfaces of different reflection phase characteristics.

operation of a thin wire dipole at a height of $0.03 \lambda$ from the ground plane. The range of useful phase angles was identified in Section II-B. The useful phase angles are indicated by two dark shaded rectangles in Fig. 7 and are satisfied by the ideal phase curve. In contrast, the four adjacent curves contain only a small portion of the useful phase angles. The resulting VSWR characteristics that correspond to each reflection phase curve for the thin wire dipole in consideration are shown in Fig. 8. Clearly only the curve corresponding to the ideal phase shows a dual band response with bandwidths of $3.6 \%$ and $5.5 \%$ along the low and high frequency bands, respectively. Note that the bandwidth of the wire dipole in free-space is $6 \%$. By observing the driving point impedance shown in Fig. 9(a) and (b) it is evident that the resistance data corresponding to the "ideal phase" curve is much

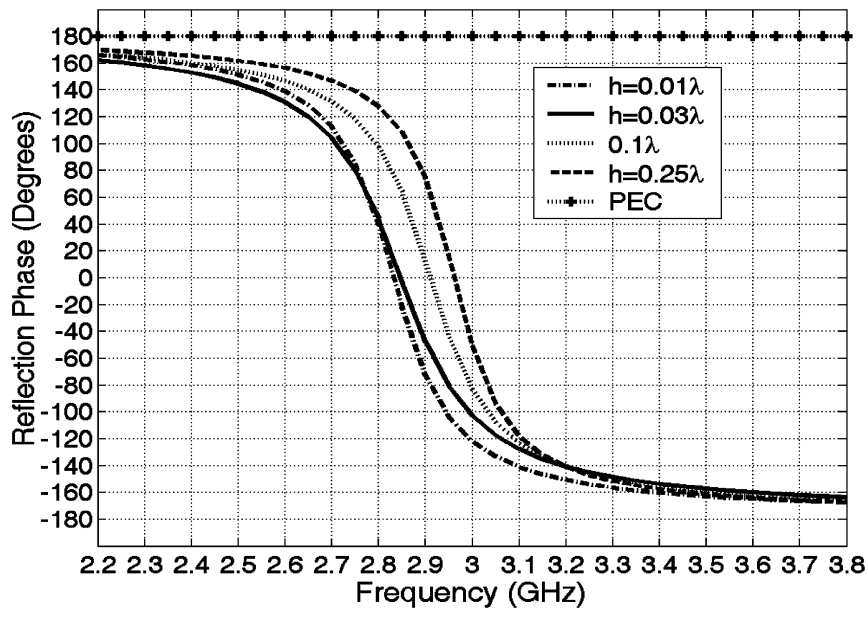

Fig. 10. Reflection phase characteristics of different EBG surfaces designed based on the height of dipole.

closer to $50 \Omega$ compared to the other four sets of data within the expected frequency bands. Similarly, the thin wire dipole was further studied for other antenna heights. For each height, an optimum reflection phase characteristic curve was generated based on our knowledge of the useful reflection phase angles. In other words, the reflection phase curve for an EBG was generated in such a manner that for a specific height of the dipole the useful range of reflection phase angles were mapped to their corresponding frequency bands by varying the parameters $a$ and $b$. The optimum reflection phase curves for different dipole heights are shown in Fig. 10. Corresponding driving-point resistance and reactance data are shown in Fig. 11. Unlike the monotonically varying resistance and reactance on a PEC, the resistance and reactance on an EBG show the signs of multiple series and parallel type resonances. For instance, for $h=0.03 \lambda$ the series, parallel, and series resonant frequencies are $2.77,2.82$, and $3.08 \mathrm{GHz}$ with driving point resistances of 70,100 , and $55 \Omega$, respectively. These resistance values along with a slowly varying reactance curve within the frequency range of interest ensure efficient dual-band operation. Similar phenomena can be observed for all other antenna heights associated with an EBG structure. The same viewpoint can be further strengthened by analyzing the VSWR data shown in Fig. 12. In contrast, the resistance for the dipole at a height of $0.1 \lambda$ from a PEC is close to $20 \Omega$ around the frequency of interest, which results in higher VSWR. However, this problem is solved when the antenna height is increased to $0.25 \lambda$ which makes the whole structure thick and bulky. 


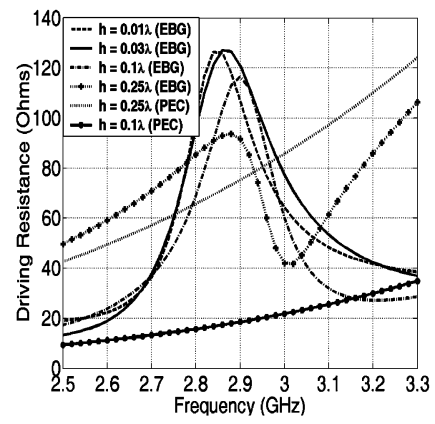

(a)

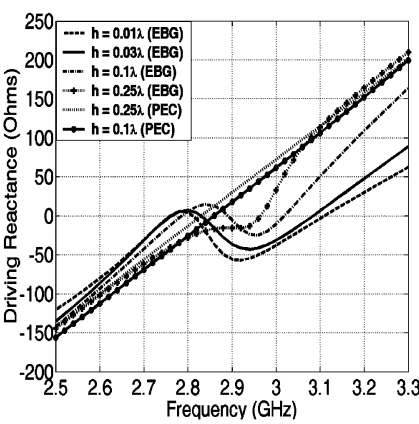

(b)
Fig. 11. Computed driving point (a) resistance and (b) reactance of a thin-wire dipole placed on top of an EBG and PEC at different heights.

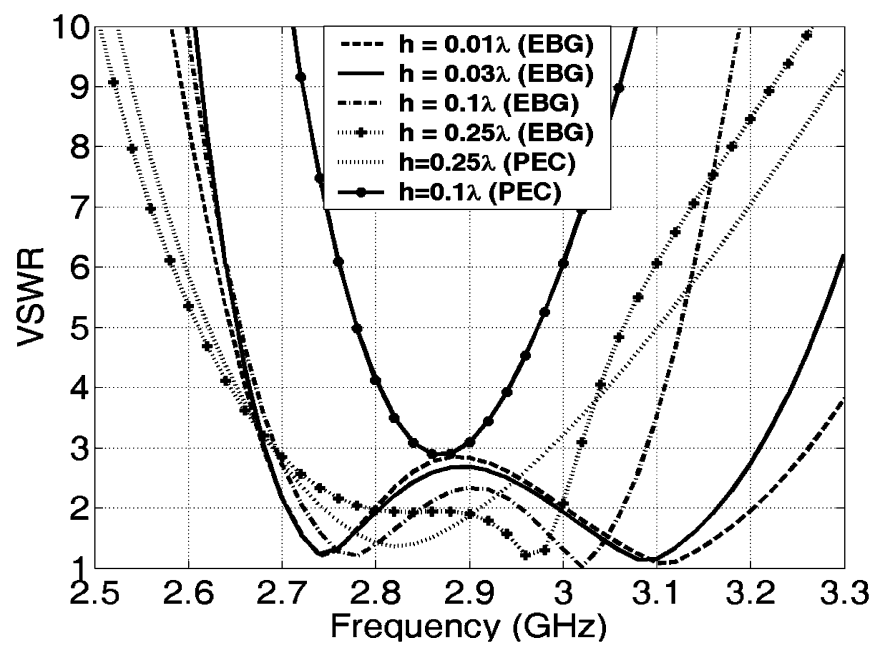

Fig. 12. Corresponding VSWR data of the thin-wire dipole.

Based on the analytical study presented, one can easily determine the optimum values of design parameters ( $a$ and $b$ ) for an EBG structure as shown in Fig. 13(a), which in turn predicts the inductance $(L)$ and capacitance $(C)$ given by [2]

$$
\begin{aligned}
& L=\mu t \\
& C=\frac{\varepsilon_{0} \varepsilon_{r} A}{d}
\end{aligned}
$$

where $t, d$ and $A$ represent the height of layer- 2 from the ground plane, gap between layer-1 and layer-2 [see Fig. 13(b)] and overlapping area between the two layers of metal plates [see Fig. 13(c)].

The steps involved in designing an EBG structure for a dipole antenna include the following. First, identify the useful range of phase angles and the corresponding frequency bands for a printed dipole of specific height considering a hypothetical EBG structure inserted between the antenna and the ground plane. Second, based on the data found in the previous step generate an optimum EBG profile that satisfies the required reflection phase angles. Third, calculate the sheet inductance, $L$ and capacitance, $C$ values from the optimum EBG design parameters $a$ and $b$. Finally, design and develop an actual EBG structure that conforms to the reflection phase requirements by evaluating the values of $t, d$ and $A$ from $L$ and $C$.

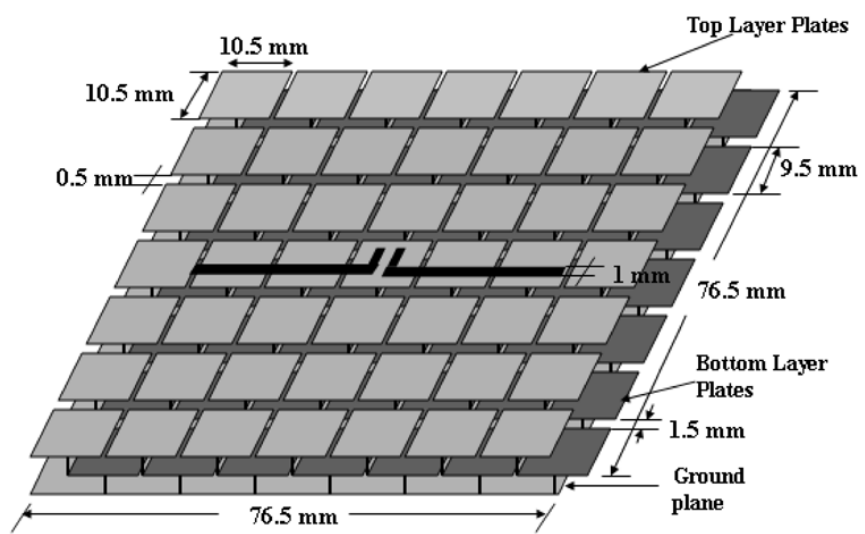

(a)

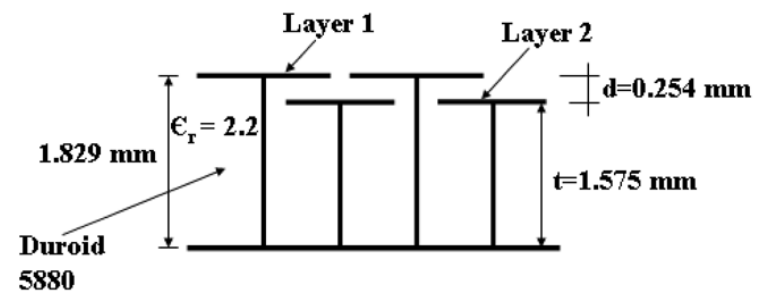

(b)

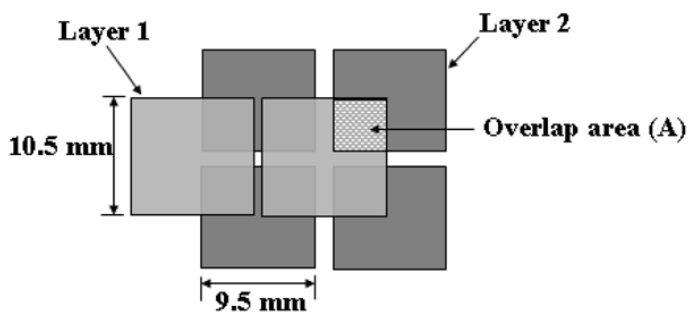

(c)

Fig. 13. (a) Schematic diagram, (b) Side view and (c) Top view of a 3-Layer EBG surface.

\section{EBG STRUCTURE DESIGN}

Considering an antenna height of $0.03 \lambda$ and based on the optimum EBG parameter values $\left(a=4.65 \times 10^{-17}\right.$ and $b=$ $\left.1.1 \times 10^{-8}\right)$ a three-layer EBG structure was designed, which is shown in Fig. 13. The vias connecting the metal plates with the ground plane provide the necessary inductance while the overlapping areas between the two layers of metal plates provide the capacitance. Although the analytical data for an antenna height of $0.01 \lambda$ (shown in Fig. 4) demonstrate good performance, for fabrication simplicity we selected an antenna height of $0.03 \lambda$. The first and second layers of metal plates (see Fig. 13) are placed at heights of 1.83 and $1.575 \mathrm{~mm}$, respectively from the ground plane. RT/Duroid $5880\left(\varepsilon_{r}=2.2\right)$ was used for both layers. A thin (strip width $=1 \mathrm{~mm}$ ) printed dipole antenna of length $45 \mathrm{~mm}$ was designed to operate at $2.9 \mathrm{GHz}$. The antenna was printed on a $0.5-\mathrm{mm}$ thick RT/Duroid 5880 substrate. Since the dipole was fed using a coaxial transmission line a lumped element balun was also designed as described in [24]. The balun circuit consisted of two capacitors $(0.92 \mathrm{pF})$ and two inductors (3.27 $\mathrm{nH})$, as shown in the circuit diagram in Fig. 14. A spacing layer (Rohacell Foam) of thickness $0.5 \mathrm{~mm}$ was inserted in between the EBG structure and the dipole. Thus, the total thickness of the whole EBG structure containing the dipole was $2.8 \mathrm{~mm}$. 


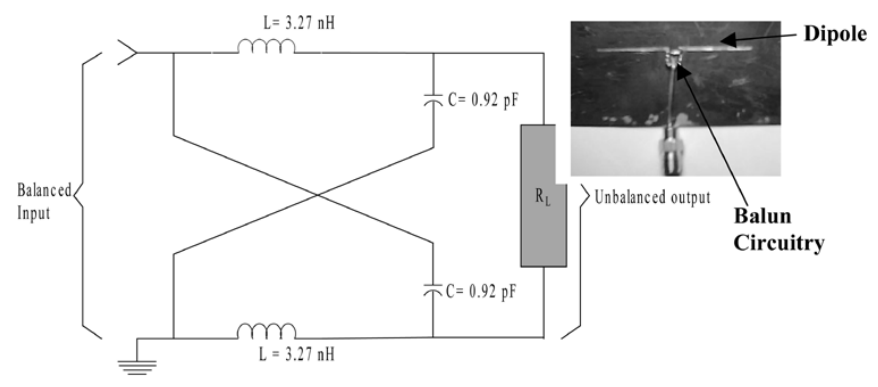

Fig. 14. Circuit diagram of the lumped element balun.

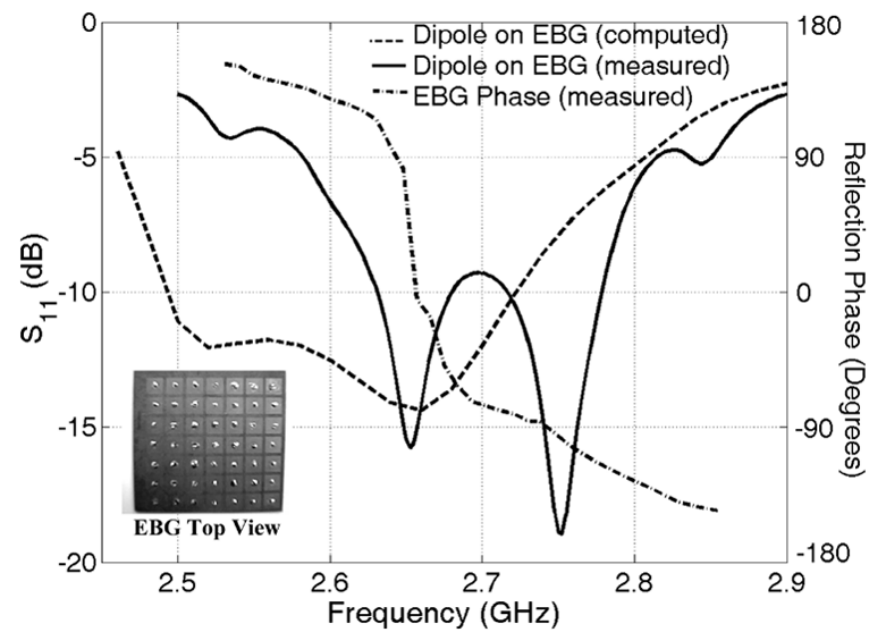

Fig. 15. $S_{11}$ Data of a dipole of strip-width of $1 \mathrm{~mm}$.

\section{EXPERIMENTAL RESULTS}

Before fabrication, the printed dipole placed on the proposed EBG structure was analyzed using IE3D [25]. Computed returnloss $\left(S_{11}\right)$ versus frequency data are shown in Fig. 15, wherefrom it is clear that the dipole has a distinct dual-band characteristics. The bandwidth of the antenna is $9 \%$ within $S_{11} \leq$ $-10 \mathrm{~dB}$. Measured $S_{11}$ data for the dipole on top of the EBG structure are plotted in Fig. 15, which again clearly show the presence of dual-band performance with bandwidth of $6 \%$. The bandwidth for the measured case is slightly narrower compared to the computed case. The discrepancies between the measured and the computed data might have been caused partly due to the approximating nature of the EBG design method. However, fabrication imperfection may also have played a role in this discrepancy. Measured reflection phase data of the proposed EBG structure is plotted in Fig. 15. Comparing these data with the phase data computed using (5), labeled "ideal phase" and shown in Fig. 7 it is clear that the measured phase crosses the $0^{\circ}$ angle at around $2.66 \mathrm{GHz}$ while the former ideal phase does it at 2.85 $\mathrm{GHz}$. The measured reflection phase curve is steeper than the ideal phase curve plotted in Fig. 7. The measured phase matches with the measured $S_{11}$ data closely( Fig. 15). The ideal phase in Fig. 7 predicts an approximate antenna bandwidth of more than $450 \mathrm{MHz}$ as illustrated in Fig. 8. The fabricated EBG structure provides a bandwidth of approximately $150 \mathrm{MHz}$ which is due to its steeper reflection phase profile.

Computed normalized radiation patterns of the dipole on the proposed three-layer EBG at 2.55 and $2.65 \mathrm{GHz}$ are shown in

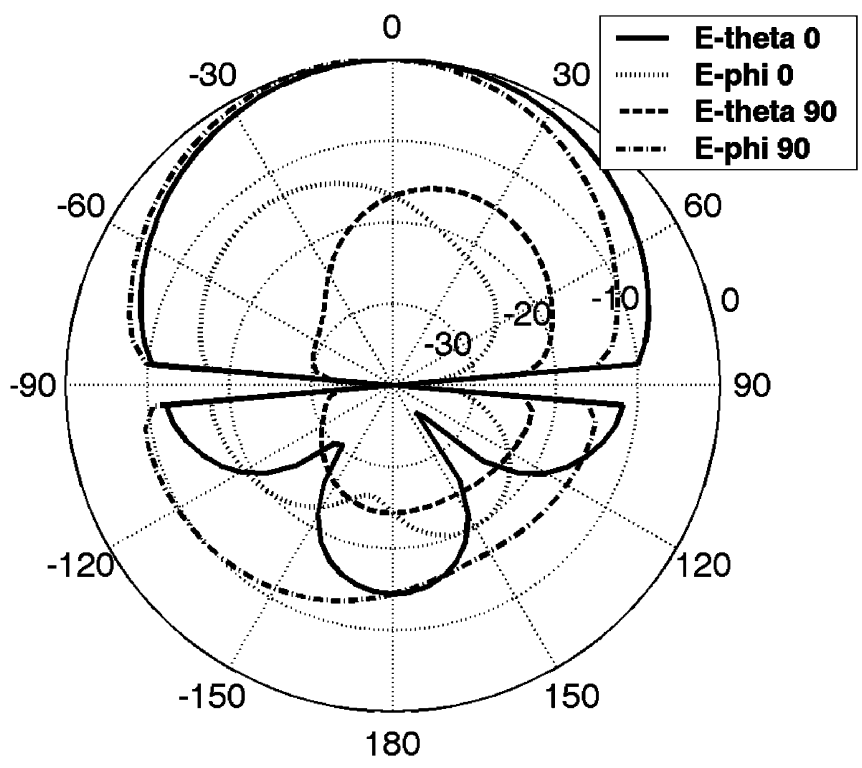

(a)

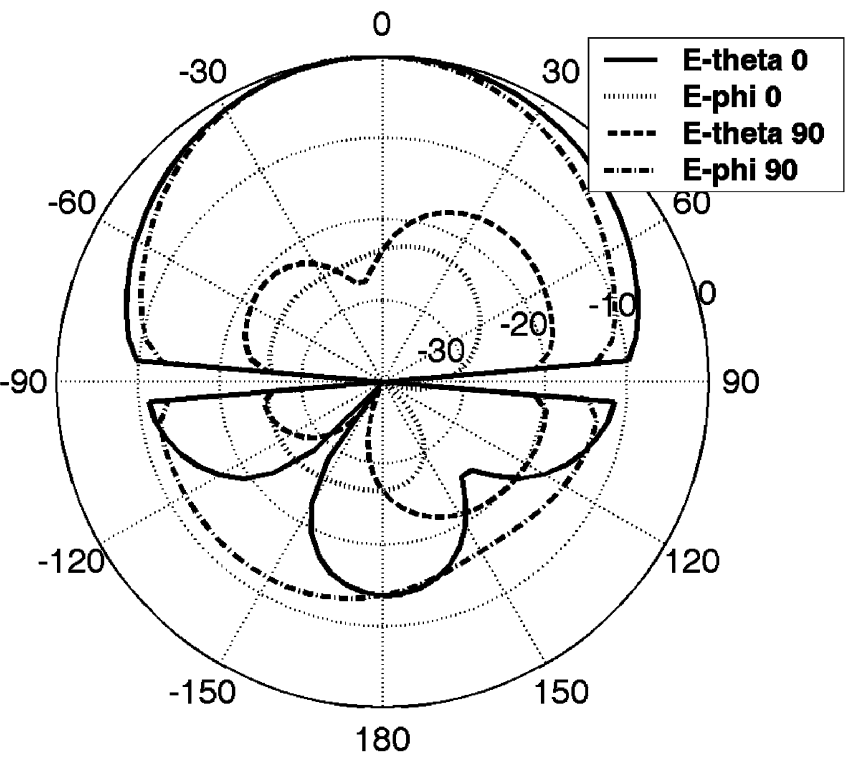

(b)

Fig. 16. Computed radiation pattern data of the dipole at (a) $2.55 \mathrm{GHz}$ and (b) $2.65 \mathrm{GHz}$.

Fig. 16. Table II lists the peak gain, directivity, cross-polarization level and front to back (F/B) ratios for five different frequency points. According to Fig. 16, the $E_{\theta}$ component in the $\phi=0^{\circ}$ plane and the $E_{\phi}$ component in the $\phi=90^{\circ}$ plane are the primary components for both the frequencies. Both the $E_{\phi}$ component in the $\phi=0^{\circ}$ plane and $E_{\theta}$ component in the $\phi=90^{\circ}$ plane are below $15 \mathrm{~dB}$ of that of their respective primary components. The radiation pattern peaks at both frequencies are along $\theta=0^{\circ}$. The peak gain of the dipole antenna at 2.55 and $2.65 \mathrm{GHz}$ are 7.0 and $7.2 \mathrm{dBi}$, respectively. Computed gain and directivity data for three other frequency points are also listed in Table II, which show similar characteristics. The beam peak directions, gain, cross-polarization and F/B ratios are good throughout the operating frequency band defined by the return loss characteristics shown in Fig. 15. 
TABLE II

Computed Gain and Directivity Data of a Dipole Placed on Top of an EBG Structure Operating at a Height of 0.03 $\lambda$ FROM THE GROUND PLANE

\begin{tabular}{|c|c|c|c|c|}
\hline $\begin{array}{c}\text { Frequency } \\
(\mathbf{G H z})\end{array}$ & $\begin{array}{c}\text { Peak Gain } \\
\text { (dBi) }\end{array}$ & $\begin{array}{c}\text { Directivity } \\
\text { (dBi) }\end{array}$ & $\begin{array}{c}\text { Cross-polarization } \\
\text { (dB) }\end{array}$ & $\begin{array}{c}\text { F/B Ratio } \\
\text { (dB) }\end{array}$ \\
\hline 2.5 & 5.5 & 5.9 & 8.0 & 14.5 \\
\hline 2.55 & 7.0 & 7.8 & 15.0 & 15.0 \\
\hline 2.6 & 7.1 & 7.3 & 15.0 & 14.0 \\
\hline 2.65 & 7.2 & 7.4 & 15.0 & 13.0 \\
\hline 2.7 & 7.1 & 7.4 & 17.0 & 12.0 \\
\hline
\end{tabular}

\section{CONCLUSION}

A comprehensive study on the effects of the reflection phase profiles of EBG structures on dipole antennas is presented. The first part of the study includes the characterization of the dipole driving-point impedance and bandwidth as function of a hypothetical EBG structure that can provide a constant reflection phase. Several interesting conclusions can be drawn from this analysis: (1) an extremely thin (antenna height equal to $0.01 \lambda$ ) dipole antenna may be realized, (2) the input reactance demonstrates a distinct dual-resonance characteristic, one for the positive and one for the negative phase angle, and (3) with increasing antenna height the dual resonance phenomenon disappears since the effect of the mutual impedance on the driven dipole starts to vanish. In the second part, we focus on studying the antenna impedance as function of various reflection phase profiles that vary with frequency, yet have the same stop-band. It is clarified that although many different reflection phase profiles can be generated that satisfy the same stop-band frequency, it is the profile that satisfies some specific reflection phase angles is required to achieve good performance. Such optimum EBG phase profiles are generated for a number of antenna heights and their influence on a dipole antenna impedance and bandwidth are studied. In the third stage, an actual EBG structure is designed and simulated along with a printed dipole antenna using IE3D. The overall antenna height for this case was $0.03 \lambda$. An experimental prototype of the said EBG structure and the printed dipole was also fabricated and tested. The computed and measured results show that significant improvement in impedance bandwidth and radiation pattern can be obtained by using an EBG structure when antenna height is very small.

The study presented in [21] considered determining the suitable reflection phase angles by varying the length of the dipole antenna and observing the antenna return loss characteristics. In contrast, the objective of this paper is to investigate the effect of the EBG reflection phase characteristics on the driving-point impedance of a dipole antenna. The present paper focuses on the intuitive, simple, analytical approach to understand the effect of EBG reflection phase on the antenna driving-point impedance. Another interesting observation here is the fact that the phase change of the EBG structure from positive to negative values result in a dual-resonance characteristic for the dipole antenna.

\section{REFERENCES}

[1] R. C. Hansen, Phased Array Antennas, New York: Wiley, 1997.

[2] D. F. Sievenpiper, "High impedance electromagnetic surfaces," Ph.D. dissertation, Univ. California at Los Angeles, 1999.
[3] H. D. Yang, N. G. Alexopoulos, and E. Yablonovitch, "Photonic band-gap materials for high-gain printed circuit antennas," IEEE Trans. Antennas Propag., vol. 45, no. 1, pp. 185-187, Jan. 1997.

[4] R. Coccioli, F. R. Yang, K. P. Ma, and T. Itoh, "Aperture-coupled patch antenna on UC-PBG substrate," IEEE Trans. Microwave Theory Tech., vol. 47, no. 11, pp. 2123-2130, Nov. 1999.

[5] J. S. Colburn and Y. Rahmat-Samii, "Patch antennas on externally perforated high dielectric constant substrates," IEEE Trans. Antennas Propag., vol. 47, no. 12, pp. 1785-1794, Dec. 1999.

[6] R. F. J. Broas, D. F. Sievenpiper, and E. Yablonovitch, "A high-impedance ground plane applied to a cell-phone handset geometry," IEEE Trans. Microwave Theory Tech., vol. 49, no. 7, pp. 1262-1265, Jul. 2001.

[7] F. Yang and Y. Rahmat-Samii, "Mutual coupling reduction of microstrip antennas using electromagnetic band-gap structure," in Proc. IEEE AP-S Int. Symp. Dig., vol. 2, Jul. 2001, pp. 478-481.

[8] M. Rahman and M. Stuchly, "Wide-band microstrip patch antenna with planar PBG structure," in Proc. IEEE AP-S Int. Symp. Dig., vol. 2, Jul. 2001, pp. 486-489.

[9] S. Sharma and L. Shafai, "Enhanced performance of an aperture-coupled rectangular microstrip antenna on a simplified uniplanar compact photonic bandgap (UCPBG) structure," in Proc. IEEE AP-S Int. Symp. Dig., vol. 2, Jul. 2001, pp. 498-501.

[10] F. Yang and Y. Rahmat-Samii, "Microstrip antennas integrated with electromagnetic band-gap (EBG) structures: A low mutual coupling design for array applications," IEEE Trans. Antennas Propag., vol. 51, no. 10, pp. 2939-2949, Oct. 2003.

[11] R. Gonzalo, P. de Maagt, and M. Sorolla, "Enhanced patch-antenna performance by suppressing surface waves using photonic-bandgap substrates," IEEE Trans. Microwave Theory Tech., vol. 47, no. 11, pp. 2131-2138, Nov. 1999.

[12] D. Sievenpiper, J. Schaffner, B. Loo, G. Tangonan, R. Harold, J. Pikulski, and R. Garcia, "Electronic beam steering using a varactortuned impedance surface," in Proc. IEEE AP-S Int. Symp. Dig., vol. 1, Jul. 2001, pp. 174-177.

[13] Y. J. Park, A. Herschlein, and P. H. Wiesbeck, "A photonic bandgap (PBG) structure for guiding and suppressing surface waves in millimeter-wave antennas," IEEE Trans. Microwave Theory Tech., vol. 49, no. 10 , pp. 1854-1859, Oct. 2001.

[14] D. Pavlickovski and R. B. Waterhouse, "Shorted microstrip antenna on a photonic bandgap substrate," IEEE Trans. Antennas Propag., vol. 51, no. 9, pp. 2472-2475, Sep. 2003.

[15] D. Sievenpiper, L. Zhang, R. F. J. Broas, N. G. Alexopolous, and E. Yablonovitch, "High impedance electromagnetic surfaces with a forbidden frequency band," IEEE Trans. Microwave Theory Tech., vol. 47, no. 11, pp. 2059-2074, Nov. 1999.

[16] Z. Li and Y. Rahmat-Samii, "PBG, PMC and PEC surface for antenna applications: A comparative study," in Proc. IEEE AP-S Int. Symp. Dig., Jul. 2000, pp. 674-677.

[17] J. Y. Park, C. C. Chang, Y. Qian, and T. Itoh, "An improved low-profile cavity-backed slot antenna loaded with 2D UC-PBG reflector," in Proc. IEEE AP-S Int. Symp. Dig., vol. 4, Jul. 2001, pp. 194-197.

[18] F. Yang and Y. Rahmat-Samii, "A low profile circularly polarized curl antenna over electromagnetic band-gap (EBG) surface," Microwave Opt. Tech. Lett., vol. 31, no. 4, pp. 478-481, Nov. 2001.

[19] R. C. Hansen, "Effects of a high-impedance screen on a dipole antenna," IEEE Antennas Wireless Propag. Lett., vol. 1, pp. 46-49, 2002.

[20] Z. Du, K. Gong, J. S. Fu, B. Gao, and Z. Feng, "A compact planar inverted-F antenna with a PBG-type ground plane for mobile communications," IEEE Trans. Veh. Technol., vol. 52, no. 3, pp. 483-489, May 2003. 
[21] F. Yang and Y. Rahmat-Samii, "Reflection phase characterizations of the EBG ground plane for low profile wire antenna applications," IEEE Trans. Antennas Propag., vol. 51, no. 10, pp. 2691-2703, Oct. 2003.

[22] C. Balanis, Antenna Theory, Analysis, and Design, 2nd ed. New York: Wiley, 1997.

[23] R. S. Elliot, Antenna Theory and Design, NJ: Prentice-Hall, 1981.

[24] P. Vizmuller, RF Design Guide: Systems, Circuits, and Equations. Norwood, MA: Artech House, 1995.

[25] IE3D User's Manual Release 9. Zeland Software Inc.. [Online]. Available: http://www.zeland.com

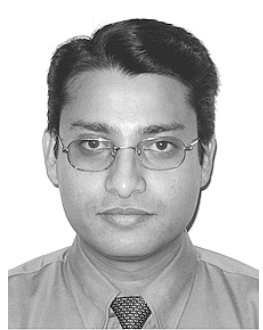

M. Faisal Abedin (S'05) was born in Dhaka, Bangladesh, in 1974. He received the B.Sc. degree in electrical engineering from the Bangladesh University of Engineering and Technology, Dhaka, in 1999, and the M.Sc. degree in electrical engineering from North Carolina State University, Raleigh, in 2001. $\mathrm{He}$ is currently working toward the Ph.D. degree in electrical engineering at the University of South Carolina, Columbia.

$\mathrm{He}$ is the author or coauthor of several refereed journal articles and conference papers. His research interests are in electromagnetic bandgap (EBG) Structures and their antenna applications, antenna miniaturization, diversity antennas, and mobile phone antennas.

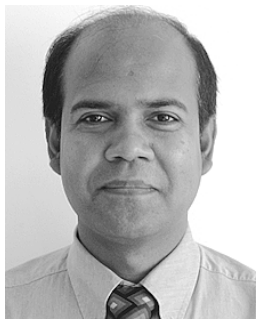

Mohammod Ali (M'93-SM'03) received the B.Sc. degree in electrical and electronic engineering from the Bangladesh University of Engineering and Technology, Dhaka, in 1987, and the M.A.Sc. and Ph.D. degrees, both in electrical engineering, from the University of Victoria, Victoria, BC, Canada, in 1994 and 1997, respectively.

He was with the Bangladesh Institute of Technology, Chittagong, from 1988 to 1992. From January 1998 to August 2001, he was with Ericsson Inc., Research Triangle Park, NC, first as a Staff Engineer and then as a Senior Staff Engineer. Since August 2001, he has been with the Department of Electrical Engineering, University of South Carolina, Columbia, where currently he is an Assistant Professor. He had also held appointments as a Visiting Research Scientist with the Motorola Corporate EME Research Laboratory, Plantation, FL, during June to August 2004. He is the author/coauthor of more than 65 publications and holds five U.S. patents. His research interests include miniaturized packaged (embedded) antennas, electromagnetic bandgap (EBG) structures and their antenna applications, fractal and multiband antennas, wireless sensors, antennas and human body interaction, and microwave circuits using wide bandgap materials.

Dr. Ali is the recipient of the 2003 National Science Foundation Faculty Career Award. 